\title{
Design and Research of the Test Data Management and Analysis System for Space Robot
}

\author{
Kai-Hang Ma ${ }^{1, a}$, Yong-Ming Gao, b ${ }^{2,}$ Lei $\mathrm{Li}^{1, \mathrm{~b}}$, Zhi-Huan $\mathrm{Wu}^{1, \mathrm{~b}}$ \\ ${ }^{1}$ Company of Postgraduate Management, Academy of Equipment, Beijing 101416, China; \\ ${ }^{2}$ Department of Information Equipment, Academy of Equipment, Beijing 101416, China. \\ a806038959@qq.com, bblackfire2008@163.com
}

Keywords: space robot, big data techniques, test data, data management, data analysis

\begin{abstract}
According to the characteristics of space robot, the test data are sorted and research on Hadoop-based data management technology, and further study for a variety of application needs space robots data analysis techniques, whereby the proposed management and analysis system test data framework, and from a holistic perspective on the data source layer, data management, data analysis layer, application layer were analyzed, and the system has been applied to verify. Practice has proved that, test data management and analysis system can solve the space robot test data management and analysis’s problems.
\end{abstract}

\section{Introduction}

Space robot is the robot that can be used in space to replace human to do experiment activities such as scientific experiment, space exploration, out of warehouse maintenance and so on, and can perform on-orbit maintenance and other operations by using the robot arm. At present, China's space robot test data management and application, compared with foreign still has a considerable gap, the traditional spacecraft test data management and analysis, mainly using a unified relational database, it is difficult to achieve distributed and balanced resource data Management mode. In addition, the deep value hidden in these test data can not be fully excavated, it is urgent to adopt appropriate and efficient analysis method. Therefore, the research on the key technology of the test data management and analysis system of the space robot can support the massive data management, rapid retrieval and analysis of space test data and operation information in the future information warfare, Monitoring, health management and fault diagnosis and forecasting applications to provide assurance.

There has some Research on the Data Management and Analysis of Orbiting Spacecraft at Home and Abroad. Paper[1] In order to solve the test data analysis and management issues, build based on B / S software architecture and "rich" client technology system; Newtera TDM of Newtera is a commercial trial data management system that can efficiently and intuitively manage test data. The main technology is Workflow Studio, which provides users with a graphical design view; paper [2] according to the experimental data system requirements analysis program, the system function module is analyzed and studied; Paper[3] a large-scale telemetry database system is designed and implemented for data management in launch vehicle test; Paper[4] aiming at the integrated management of satellite telemetry parameters, this problem is solved by using common telemetry parameters; Paper[5] according to the characteristics of telemetry parameters of missile test, a data processing platform is constructed; Paper[6] The design and implementation of data management software which based on MS SQL Server 2000 database, can be more effective management of satellite data.

\section{The Change Rule of Test Data}

Space robot with the orbit operation designed a large number of telemetry data, in order to fully reflect the performance during the working state. These telemetry data are the basis and support for the ground staff to carry on the operation management, the fault prediction, the fault diagnosis. 
Telemetry data which are analog and digital variables including volt age, pressure, count, temperature, etc; some of its changes by the larger space environment, and some variables directly related to the state of the satellite that showing a changeable law. Before the management and analysis of the Test data, it is necessary to understand the characteristics of the space robot telemetry data, study the variation rules of the satellite data, analyze the characteristics of the change and classify accordingly, and then carry on the related management and analysis.

\subsection{The characteristic of Space Robot .}

The spatial environment of the space robot and the characteristics of the executed tasks make the space robot different from other robots. There are specific performance in the following area:

(1)High environmental adaptability. The space environment mainly includes: the launch of the mechanical environment, orbit microgravity, space radiation, ultra-vacuum, etc.

(2)Complex conditions. Space robots need to meet mission requirements in different conditions that make the system design constraints more difficult.

(3)Long service life. The space robot needs to operate under the condition of no maintenance, and the service life of the space robot can be realized under the condition of complex environment.

(4)Difficult ground verification. Space robots are difficult to verify due to their complex spatial environment, ambiguous scene, and diverse tasks.

\subsection{Classification of Test Data.}

From the point of view of data analysis and data processing, telemetry data can be divided into integer, floating-point type, etc.

Integer telemetry parameters generally include identification words, status words, etc. The identification word includes the frame synchronization word, the satellite identification word etc. It is fixed binary code, usually in the form of hexadecimal, for the whole life. The status word reflects the working status of the star device that according to influence of specific circumstances, subject to satellite status and ground command control etc.

Floating-point telemetry parameters generally include voltage, current, temperature and other continuous variables, the temperature variable is mainly affected by the equipment working conditions and other factors; The voltage is divided into Primary power supply voltage and The secondary power supply voltage that both are stable, especially the secondary power supply voltage; The performance of current reflect the power consumption of the star device, with the equipment on-off machine will have a certain state of the fluctuations.

Space robot in orbit is an area that generates massive data, and it is also an area that needs the support of Big Data technology[7]. The life cycle of space robot includes large amount of data, which can be generated from the design, development, launch stage and in orbit. Figure 1 shows the data classification of an orbiting space robot.

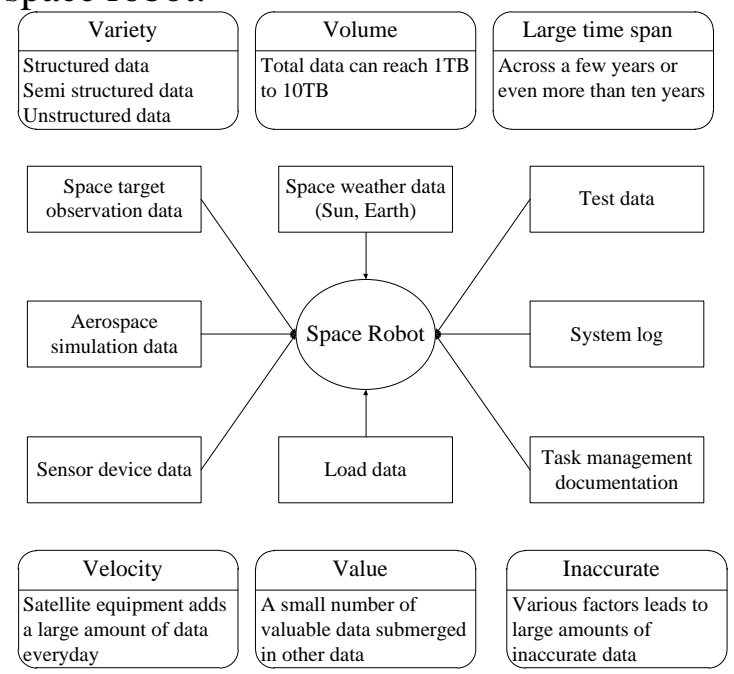

Fig. 1 Classification and characteristics of space robot test data 


\section{Research on Test Data Management and Analysis Methods}

According to the characteristics of space robot and the classification of test data in the previous section, the data management technology based on Hadoop is used to store and manage large data. Based on data management, this paper implements data preprocessing work including the analysis of mean, variance, correlation and so on. For a variety of applications of space robots, the data analysis techniques include: analysis technology for life and performance prediction of space robots, analysis technology for space robot fault diagnosis and analysis technology for space robot key technology evaluation.

\subsection{Data Management Technology Based on Hadoop.}

In 2004, Google published three papers about core technology distributed computing system: Distributed File System GFS, GFS-based data storage systems, distributed computing framework MapReduce[8]. Google's distributed computing system simplify the system of distributed computing theory, to a large extent, to reduce the difficulty of the technology to achieve, and enhance the overall capacity, just add the cost of very cheap computing equipment. After 10 years of development, Hadoop ecosystem gradually be rich, has now become the de facto international standard for distributed computing systems.

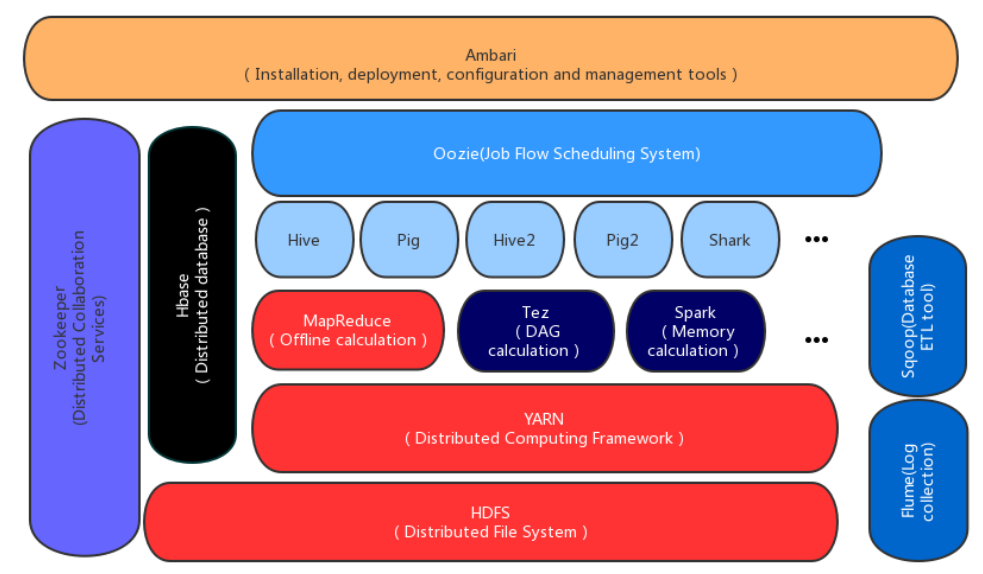

Fig. 2 Apache hadoop ecosystem

Hadoop mainly consists of three parts: MapReduce distributed processing framework, HDFS distributed file system, HBase distributed column database system. MapReduce is a distributed processing framework model, divided into two steps, map (mapping) and reduce (simplified). HDFS is a distributed file system for HBase and other tools to provide storage mechanism. HBase which is a NoSQL data can use the advantages of distributed database to read / write data quickly. Hadoop has the following characteristics:

(1)Data distributed storage. Backing up multiple copies of data on a cluster to improve reliability and availability. Users do not care about data storage location, thus eliminating the broadband bottleneck problem.

(2)Hadoop hides the complex distributed implementation process and provides a simple programming approach that provides powerful data analysis mechanisms.

(3)Huge Storage. Hadoop enables applications to run on thousands of computers and petabytes of data. In a cluster, hundreds of "small" computers have aggregate computing power that can exceed the computing power of a supercomputer and that is cheap.

(4)Distributed processing and fast data access. The I/O performance requirements are high When Cluster model is in the creation of shared data. Hadoop applications process data in an orderly fashion that can avoids random data access (disk seek operations) and mitigates I/O load.

(5)Reliability, failover and extensibility. Hadoop enhances scalability by monitoring node failures and re-performing tasks with different nodes, enabling seamless integration of multiple servers into one cluster. 


\subsection{Data Analysis Methods for Multiple Applications.}

\subsubsection{Analytical Techniques for Life and Performance Prediction of Space Robot.}

During the on orbit operation of the space robot, the change and the state of the spacecraft are monitored by the change of the telemetry data to analyze and study the trend and performance of the spacecraft components. Due to the space environment and mission capability of the space robot, the telemetry data mainly showed three kinds of characteristics: Trend, Seasonality, and Randomness. It can be decomposed first by X-11 data decomposition method, and then the decomposed data be analyzed and studied by BP neural network, nonparametric regression, ARMA model and polynomial fitting method[9]. Finally we merged the results of prediction. As shown in Figure 3.

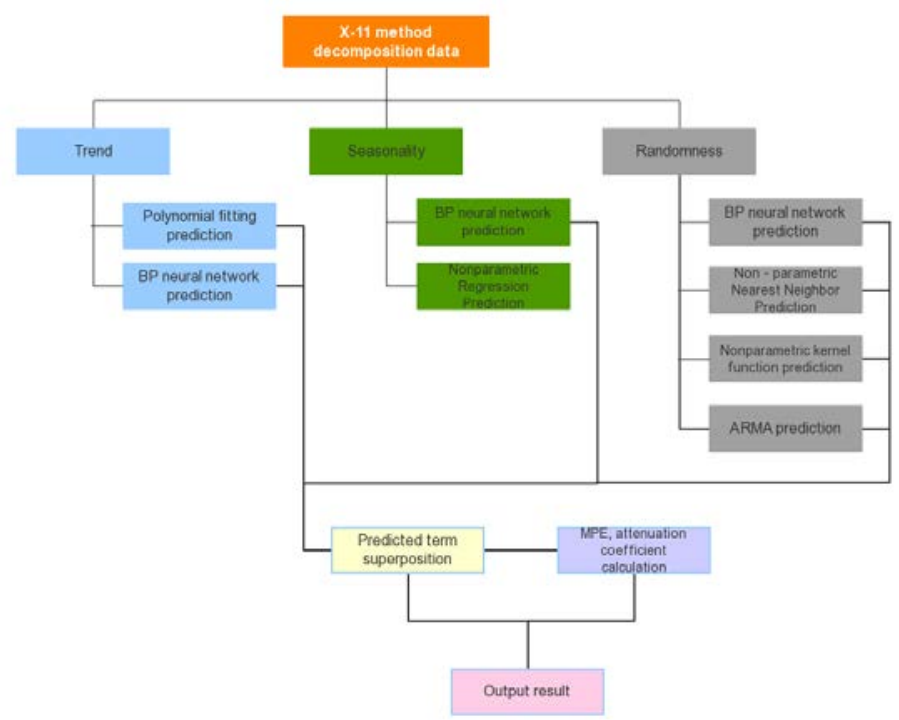

Fig. 3 Space robot performance prediction process

\subsubsection{Analysis Technique for Fault Diagnosis of Space.}

The analysis technology of space robot-oriented fault diagnosis mainly uses neural network and expert system. The system has a unified expression of internal knowledge and solve the problem of diagnosis of reasoning, knowledge acquisition and other issues in traditional expert system to some extent. Knowledge weights are stored in each connection weight, and the knowledge base can be organized and managed with large knowledge capacity. Figure 4 shows the block diagram of the diagnostic system.

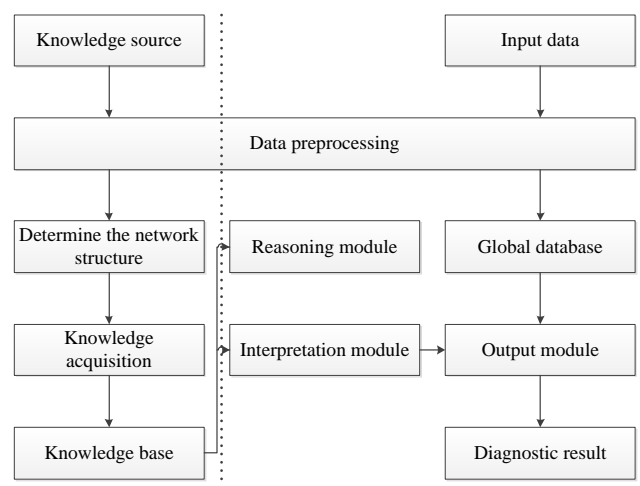

Fig. 4 Neural network expert system block diagram

The left part of the dotted line is the process that the system acquires knowledge. The knowledge base stores the knowledge acquired through the neural network and the main phenotypic form is the matrix. The right part is the fault diagnosis subsystem, the main task is to complete the input data processing, and then the data will be reasoned and the application of reasoning mechanism to get the final diagnosis. 
3.2.3 Analytical Techniques for Key Techniques evaluation of Space Robots.

After space robot's launch, when it is in-orbit operation, the relevant key technologies will verify the test by its on-board data. We mainly take the statistical analysis methods, such as regression analysis, Cluster analysis, Discriminant analysis, Principal component analysis, Neural Networks etc.

(1) regression analysis. A statistical method of studying the interdependence of two or more variables. According to the dependence of the established variables, the method derives the inherent laws. The general regression analysis include: Univariate Regression and Multiple Regression[10].

(2) Cluster analysis. it's the initial and exploration scientific research work that one of the basic methods, and so-called cluster is to study the degree of affinity between the objects of each other.

(3) Principal component analysis. Principal component analysis is in a number of variables that analyze and identify the main impact variables.

(4) Discriminant analysis. Discriminant analysis is in the case of a known type with the identified variables to divide variables into several known types.

(5) Neural Networks. It is analogous to the biological nervous system: dendrites, axons, and cell bodies. Artificial neural network algorithm is one of the most commonly used methods that can be used in a number of areas.

\section{Design of Test Data Management and Analysis Framework}

The management and analysis system based on space robots test data can receive the satellite data in real time, and can query the historical data and output the preprocessed data to the data analysis module, so as to realize the multiple application analysis of the space robot. Aiming at the management and analysis requirements of space robots experimental data, a system framework is designed and proposed, which includes application layer, data analysis layer, data management layer and data source layer. Some of the data processing functions and code implementations are reflected in the data analysis layer, and some methods and theories of the data management layer support data analysis. Figure 5 is the space robot test data management and analysis framework.

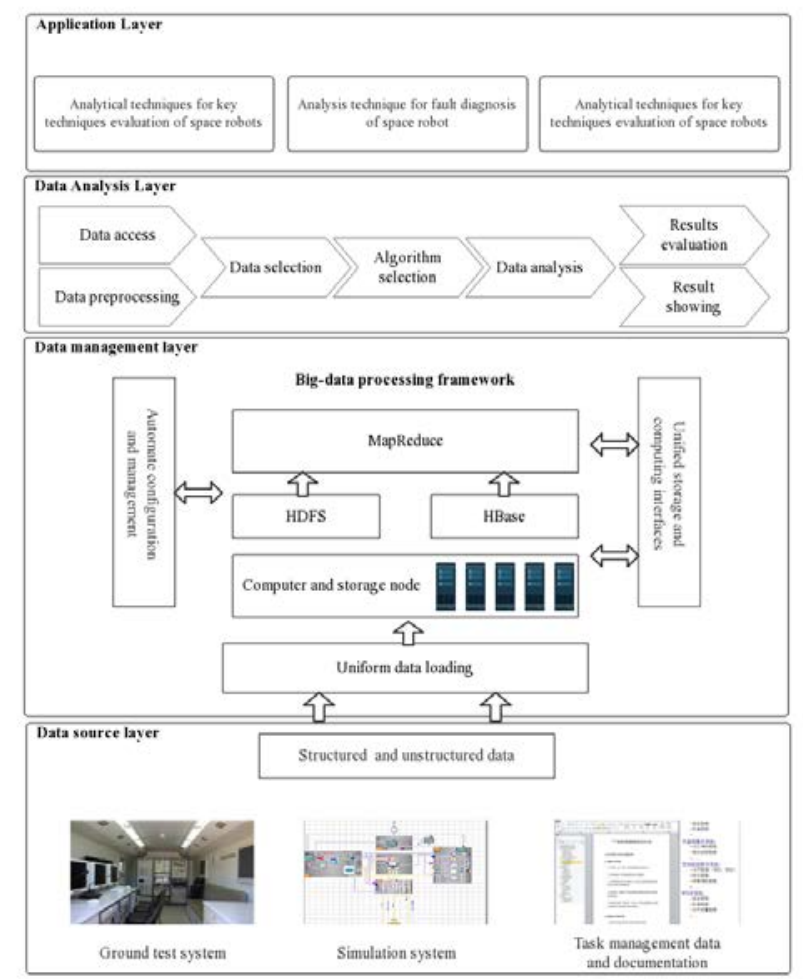

Fig. 5 Space robot test data management and analysis framework

\subsection{Data source layer}

The data source provides test data that needs to be managed and analyzed that mainly comes from the ground test system, the simulation system, the task management data and the document. A series 
of tasks from the design, development, ground simulation test, launch, space test, to orbit operation management will generate a good variety of data of space control system. Part of the space robot parts data shown in Table 1.

Table 1 Partial data of a mechanical arm subsystem

\begin{tabular}{cccccc}
\hline $\begin{array}{c}\text { System } \\
\text { time }\end{array}$ & $\begin{array}{c}\text { Bus } \\
\text { voltage }\end{array}$ & $\begin{array}{c}+ \text { X windsurfing } \\
\text { current }\end{array}$ & $\begin{array}{c}-Z \text { windsurfing } \\
\text { current }\end{array}$ & $\begin{array}{c}+ \text { Y Solar } \\
\text { current }\end{array}$ & $\begin{array}{c}+ \text { Z Solar } \\
\text { current }\end{array}$ \\
\hline 3862029 & 16.037 & 1.00345 & 0.541413 & 0.010653 & 0.03798 \\
3862039 & 16.037 & 1.01863 & 0.539568 & 0.011039 & 0.038361 \\
3862058 & 16.037 & 1.01563 & 0.534977 & 0.011039 & 0.038361 \\
3862069 & 16.0658 & 1.02297 & 0.537123 & 0.011039 & 0.03798 \\
3862078 & 16.037 & 1.00996 & 0.532832 & 0.011039 & 0.038742 \\
3862089 & 16.0658 & 1.01213 & 0.534977 & 0.011425 & 0.03856 \\
\hline
\end{tabular}

\subsection{Data management layer}

The main function of data management layer is to complete the data pre-processing and storage, with Hadoop large data management technology we can achieve in-orbit data management, data query, database query and other related operations.

(1)Data management environment configuration

The data management layer mainly completes the data storage management that is achieved by Hadoop data management technology .Hadoop installation mode should select the full distribution mode, including four Dell Optiplex990 host and a router. Cluster topology diagram shown in Figure 6.

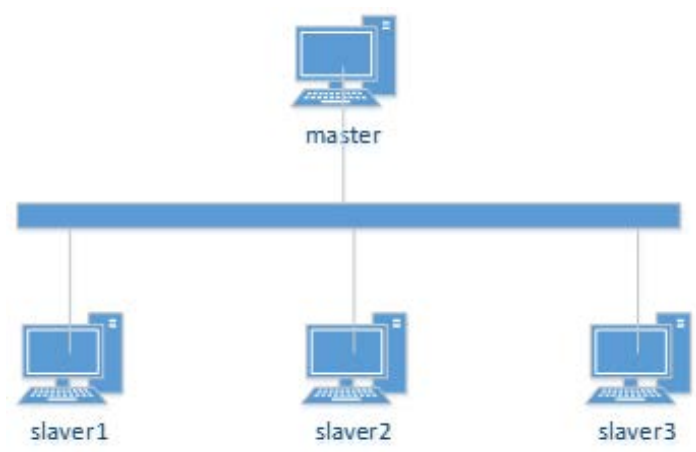

(2) Data management

Fig. 6 Hadoop cluster topology

According to the requirements of space robot test management, the data management module functions mainly include the following contents: in-orbit data management, general functional requirements of query, data query, non-telemetry data management, management query function.

\section{3 data analysis layer}

(1)Design of Data Analysis Component Based on Double-layer Architecture

Aiming at the multiple needs of Application layer, we designed the data analysis component including the basic analysis methods and advanced development methods by analyzing the data management mode and the characteristic of the test data[11].

The two-tier data analysis component integrates multiple analytical methods. The basic method layer includes a variety of statistical analysis methods, such as regression analysis, clustering analysis, principal component analysis; advanced expansion layer is a special application requirement for the integration of extended analysis algorithm is above statistical analysis methods.

(2) Functional design of data analysis layer

Data analysis layer is mainly to deal with data management layer of test data which is divided into four steps, data storage, data preprocessing, data analysis, the results of expression and display that are implemented by using components.

\subsection{Application layer}

Application layer is a user-oriented application requirements, including space robot key technology test evaluation, space robot fault diagnosis, space robot in orbit state monitoring, health 
management and performance prediction applications. Application layer is the top layer of the framework of test data management and analysis platform that mainly is the application value of user's requirements; from the top down, the application requirements of the application layer, which determines the use of space-oriented robot data analysis technology, including key technologies Assessment, fault diagnosis, health management and performance prediction; The required data management technology, which is the need for Hadoop data management technology and some pre-processing technology to meet the upper data analysis requirements; the bottom of the data source layer is the basis of the entire framework. From the bottom up, multi-type, massive data and decision Hadoop data management technology is to support a variety of data for space robot analysis technology and support user application requirements

\section{Application validation}

The application results of the management and analysis platform of space robots test data are validated by the actual data of a solar panel in an orbiting space robot. Data management and analysis platform is established, can well support a variety of user-oriented application requirements, allowing users to grasp and understand the operation of the satellite. Figure 7 shows the HBase and MapReduce implementation information when we built a large data platform. The experimental data management and analysis platform and the analysis technology for the life and performance prediction of space robots are shown in Figures 8 and 9.

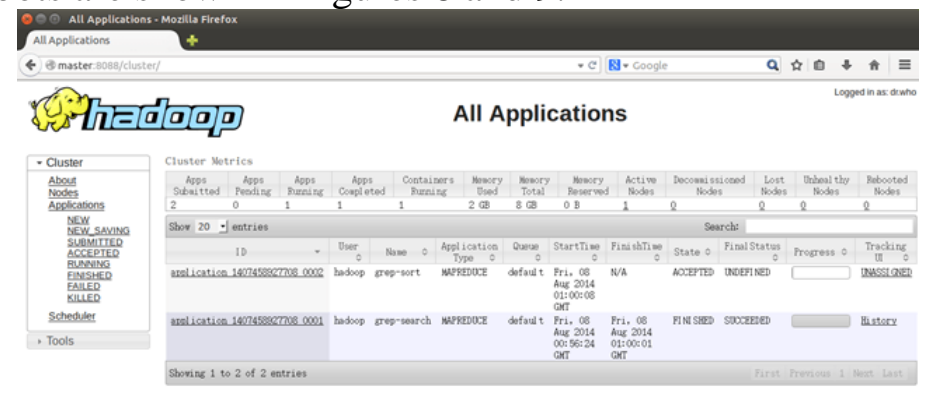

Fig. 7 HBase and mapreduce operation information

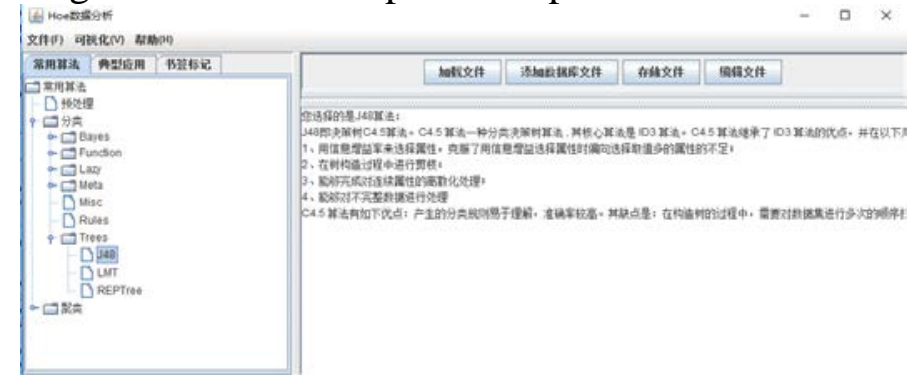

Fig. 8 Test data analysis module

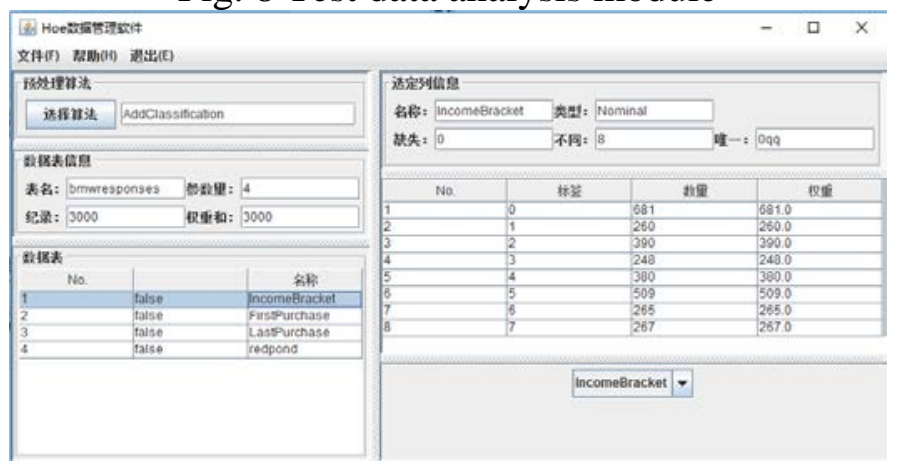

Fig. 9 Test data management module 


\section{Summary}

This paper first analyzes the characteristics of space robot task, and the test data are classified and analyzed. We combined with the application requirements, data management and data analysis technology of space robot to study the related key technologies. The test data management and analysis system which is suitable for the characteristics of the space robot is designed and implemented. The results show that the application results show that the system is effective, and the designed system can effectively manage and analyze the experimental data of space robot, and it is very important to improve the on-orbit data management and analysis of space robots.

\section{References}

[1] Wang Xiaobing, Gu Wei. The Design and Research of the Test Data Analysis and Management System for Aerospace Product. Aerospace Control,2012, 30(4):68-72.

[2] Ding Li, An Haijun. Requirement and Realization of Test Data Management System. Aeronautical Computing Technique.2010, 40(3):96-98.

[3] Nie Rongmei, Zheng Dongsheng, Luo Jun. Design Method for a Large-scale Test Database System of launch Vehicle. Missiles and Space Vehicles.2008, (1):23-28.

[4] Zheng Daixun. Design of Satellite Telemetry and Remote Control Database. Spacecraft Engineering. 2000, 9(1): 49-61.

[5] Yue Tielin. Research on General Telemetry Data Processing Platform and Exterior Ballistic Estimation Methods. Harbin Engineering University. 2012.

[6] Teng Yuandao. A thesis Submitted in Partial Fulfillment of the Requirements for the Degree of Master of Engineering. Huazhong University of Science and Technology. 2004.

[7] Wang Shan, Wang Huiju, Tan Xiongpai, et al. Architecting Big Data:Challenges,Studies and Forecasts. Chinese Journal of Computers.2011, 10:1741-1752.

[8] Maniar K B , Khatri C B.Data Science:Bigtable,Mapreduce and Google File System. International Journal of Computer Trends and Technology(IJCTT),2014,16(03):115-118

[9] Qin Wei,Guo Yongfu. A Failure Warning System Based on Historical Telemetry Data for Satellites on Orbit. Spacecraft Engineering. 2010, 19(6):40-45.

[10] Bao Yanke. Data Analysis Tutorial.Beijing:Tinghua University Press.1011:184.

[11] Huo Liping, Yang Chunying, Qiu Zhi. Design for Software Platform of ATS Based on Signal-Oriented. Measurement \& Control Technology.2005, 24(2):52-54. 